\title{
Avaliação da tecnologia Wiki: ferramenta para acesso à informação sobre ventilação mecânica em Terapia Intensiva
}

\author{
Assessment of Wiki technology: a tool for accessing information on mechanical \\ ventilation in Intensive Care \\ Evaluación de la tecnología Wiki: una herramienta para acceder a la información sobre \\ la ventilación mecánica en Cuidados Intensivos

\section{Daniela Couto Carvalho Barra', Grace Teresinha Marcon Dal Sasso", Cleusa Rios Martins", Sayonara de Fátima Faria Barbosa"}

\begin{abstract}
' Universidade Federal de Santa Catarina, Departamento de Enfermagem, Programa de Pós-Graduação em Enfermagem (Doutoranda), Grupo de Pesquisa em Tecnologias, Informações e Informática em Saúde e Enfermagem. Florianópolis-SC, Brasil.

"Universidade Federal de Santa Catarina, Departamento de Enfermagem, Programa de Pós-Graduação em Enfermagem (Doutoranda), Grupo de Pesquisa em Tecnologias, Informações e Informática em Saúde e Enfermagem. Florianópolis-SC, Brasil.
\end{abstract}

Submissão: 24-05-2010 Aprovação: 23-07-2012

\begin{abstract}
RESUMO
O desenvolvimento e a aplicabilidade da informática influenciam todas as áreas do conhecimento, possibilitando novas formas de aprendizagem. O Wiki é uma ferramenta da informação e comunicação disponibilizada pela tecnologia da WEB 2.0 que pode ser explorada e utilizada no ensino, aprendizagem, cuidado e na pesquisa na área da Enfermagem. Assim, este estudo, de natureza quantitativa, do tipo descritivo e exploratório, objetivou avaliar com os acadêmicos de enfermagem os critérios de Ergonomia e Usabilidade da ferramenta Wiki como tecnologia de acesso à informação sobre os cuidados de enfermagem em ventilação mecânica na Unidade de Terapia Intensiva. A ferramenta foi avaliada como "excelente" quanto aos critérios de Ergonomia e Usabilidade, sendo considerada uma nova tecnologia emergente apropriada para o uso educacional.

Descritores: Informática em Enfermagem; Educação em Enfermagem; Tecnologia Educacional; Cuidados de Enfermagem; Unidades de Terapia Intensiva.
\end{abstract}

\begin{abstract}
The development and application of information technology influence all areas of knowledge, enabling new ways of learning. The Wiki is a tool of information and communication technology provided by the Web 2.0 that can be exploited and used in teaching, learning, care and research in nursing education. Thus, this quantitative study is a descriptive and exploratory objective was to evaluate the nursing students with the criteria of Ergonomics and Usability of the tool Wiki as a technology to access information on nursing care in mechanical ventilation in the Intensive Care Unit. The tool was evaluated as "excellent" in the criteria for Ergonomics and Usability, and is considered a new emerging technology suitable for educational use.

Key words: Nursing Informatics; Nursing Education; Educational Technology; Nursing Care; Intensive Care Units.
\end{abstract}

\section{RESUMEN}

El desarrollo y aplicación de tecnología de la información influyen en todas las áreas del conocimiento, lo que permite nuevas formas de aprendizaje. El Wiki es una herramienta de información y tecnología de comunicación proporcionados por la Web 2.0, que puede ser explotada y utilizada en la enseñanza, el aprendizaje, la atención y la investigación en educación en Enfermería. Así, este estudio cuantitativo, descriptivo y exploratorio, objetivó evaluar, junto a los estudiantes de enfermería, los criterios de Ergonomía y Usabilidad de la herramienta Wiki, como una tecnología para acceder a la información sobre los cuidados de enfermería en ventilación mecánica en la Unidad de Cuidados Intensivos. La herramienta fue evaluada como "excelente" en los criterios de Ergonomía y Usabilidad, siendo considerada una nueva tecnología adecuada para el uso educativo.

Palabras clave: Informática Aplicada a la Enfermería; Educación en Enfermería; Tecnología Educacional; Atención de Enfermería; Unidades de Terapia Intensiva.

\section{AUTOR CORRESPONDENTE Grace Teresinha Marcon Dal Sasso E-mail: grace@ccs.ufsc.br}




\section{INTRODUÇÃO}

O cuidado de Enfermagem, especialmente em Unidades de Terapia Intensiva (UTI), é complexo e desafiador. Os enfermeiros estão regularmente expostos a situações clínicas difíceis que requerem atenção e controle e, ao mesmo tempo, a inovações tecnológicas que precisam estar integradas de forma consistente, correta e segura ao sistema de cuidado à beira do leito. Cada tecnologia adicionada ao cuidado e ao sistema organizacional aumenta a complexidade do trabalho da Enfermagem embora, também, potencialize a redução da carga de trabalho, melhorando a qualidade do cuidado e diminuindo os erros e eventos adversos ${ }^{(1)}$.

A partir do cenário delineado, entende-se que a informática e os sistemas de informação estão cada vez mais disponíveis para apoiar a prática, a educação, a pesquisa, o cuidado e o desenvolvimento político, social e econômico da profissão de Enfermagem.

Neste sentido, observa-se que o desenvolvimento e a aplicabilidade da informática influenciam todas as áreas do conhecimento, através da difusão de informações, criação de programas de ensino e geração de controvérsias, possibilitando, assim, novas formas de aprendizagem. A Enfermagem, sendo uma profissão social, pode encontrar na informática os subsídios para incorporar novas formas de construção do saber, bem como nas formas de produção, de gestão e de disseminação do conhecimento e das informações ${ }^{(2)}$.

A aliança entre a Educação em Enfermagem e a Informática em Enfermagem pode ser interpretada como: 1) fenômeno social que transforma a vida dos indivíduos, grupos e comunidades; 2) amplificador intelectual que fundamentado em métodos pedagógicos de análise e em linguagens simbólicas ou não promovem uma nova representação do conhecimento em Enfermagem, ou seja, uma nova interpretação de problemas da profissão que envolve atividades cognitivas; 3 ) fonte de recursos instrumentais em condições de enfrentar e equacionar questões de naturezas diversas, desde que utilizados de modo adequado e coerente com os objetivos que a profissão deseja alcançar(2).

Nos últimos anos se vivencia um novo tipo de gestão social do conhecimento, que se apoia num modelo interativo, onde o aluno é produtor do seu próprio processo de ensino-aprendizagem. Novas formas de abordagem educacional devem ser mais atualizadas e coerentes com as novas demandas da sociedade. Para alcançar uma educação utilizando o computador, que seja construtiva e controlada pelo aluno, bem como, para desenvolver e provar seus próprios modelos de pensamentos críticos, o educador deverá utilizar uma série de estratégias que promovam a capacidade de autogestão do ato de aprender, proporcionando ao aluno a oportunidade de percorrer por si mesmo o caminho de ambientes educativos ricos e criativos, com propósitos claros e bem definidos ${ }^{(2-4)}$.

Nesta perspectiva, os pressupostos do construtivismo, fundamentados na concepção do aprendiz como o construtor do seu próprio conhecimento ${ }^{(5)}$, aliados às tecnologias disponibilizadas pela informática, colocam o aluno como responsável pela elaboração de conhecimentos e crenças a partir dos seus conhecimentos preexistentes. Este uso construtivista da tecnologia propicia aos alunos a construção de conhecimentos complexos e pessoalmente significativos ${ }^{(6-7)}$.

Visando adotar novas estratégias educacionais para o acesso à informação no processo de ensino-aprendizagem referente aos cuidados de Enfermagem para os pacientes assistidos pela ventilação mecânica no ambiente das Unidades de Terapia Intensiva (UTIs), fundamentados nos pressupostos construtivistas, entende-se que as tecnologias da Web 2.0 são as ferramentas pedagógicas mais atualizadas e disponíveis voltadas para a construção do conhecimento na área da Enfermagem.

Especialmente na área da saúde, a Web 2.0 é uma nova tecnologia emergente que, por meio da interação coletiva, as pessoas/usuários podem colaborar para a qualidade do conteúdo disponível, mediante a produção, classificação e reformulação do que já está disponível. Em síntese, a Web 2.0 é uma nova concepção, que passa a ser descentralizada e onde o usuário torna-se um participante ativo sobre a criação e seleção do conteúdo postado em um determinado site através de plataformas abertas e dinâmicas. Nela, os arquivos ficam disponíveis on-line, podendo ser acessados em qualquer lugar e momento. Ou seja, torna-se possível criar uma conexão entre usuários com interesses comuns utilizando a internet, onde as pessoas reformulam e criam novos conteúdos ${ }^{(8)}$.

A tecnologia Web 2.0 disponibiliza diversas ferramentas de comunicação e informação que podem ser exploradas e utilizadas no ensino, na aprendizagem, no cuidado e na pesquisa na área da Enfermagem. Entre elas destacam-se: Wiki, blogs, e-portifólios, podcasting, redes sociais, tecnologias portáteis, coursewares, realidade virtual, tecnologias de gerenciamento da informação pessoal, entre outras.

Um dos principais expoentes desta nova tecnologia emergente são os Wikis. O Wiki é definido como um sistema livremente expansível de páginas web interligadas num sistema de hipertexto para armazenar e modificar informação - um banco de dados, onde cada página é facilmente editada por qualquer usuário com um navegador de internet/browser ${ }^{(11)}$ (exemplos: Microsoft Internet Explorer; Firefox; ${ }^{\circledR}$ Google Chrome ${ }^{\circledR}$ ). Sua estrutura foi produzida visando oferecer uma integração do conhecimento de diversas áreas por meio de associações variadas, inclusive fatores socioculturais, linguísticos e acadêmicos. Esta organização consolidou-se na web assumindo uma reprodução dinâmica de si mesma com o crescimento de usuários colaboradores. Os novos links inseridos são atualizados periodicamente pelos usuários, conforme as mudanças que ocorrem na própria web, desse modo adicionando novos conhecimentos e removendo ou alterando os conteúdos já existentes ${ }^{(8)}$.

Quando se menciona a utilização de novas estratégias de ensino para o acesso à informação, acredita-se que a ferramenta Wiki, por meio da sua interatividade e flexibilidade, seja uma tecnologia bastante apropriada para o uso educacional ${ }^{(8)}$. Os Wikis podem ser utilizados sob diversas formas para auxiliar os processos de ensino-aprendizagem, tais como: a) o professor pode enviar alguns termos-chave para que os alunos possam desenvolvê-los na edição de texto; b) os alunos podem trabalhar em grupos, editando textos de forma colaborativa; c) os alunos podem adicionar nos Wikis os resultados de 
pesquisas realizadas, compartilhando-as com os participantes e; d) um Wiki pode ser usado como portfólio mostrando a evolução de um projeto ${ }^{(9)}$.

Neste sentido, entende-se que é indispensável desenvolver, adequar e aplicar os recursos tecnológicos disponíveis para a melhoria dos processos de educação e de trabalho de Enfermagem, avaliando se as tecnologias existentes estão sendo utilizadas corretamente e quais poderão ser desenvolvidas para propiciar melhor qualidade na formação dos recursos humanos para a prestação dos cuidados de Enfermagem, visando promover a saúde do indivíduo e de sua coletividade ${ }^{(10)}$.

Diante destas considerações, este estudo buscou integrar a tecnologia Wiki ao processo de educar-cuidar em Enfermagem em UTI, com o intuito de contribuir com a formação dos futuros enfermeiros, o desenvolvimento e a construção coletiva do conhecimento e da prática de Enfermagem. O estudo teve como objetivo avaliar com os acadêmicos de enfermagem os critérios de Ergonomia e Usabilidade da ferramenta Wiki como tecnologia de acesso à informação sobre os cuidados de enfermagem em ventilação mecânica na Unidade de Terapia Intensiva.

\section{MÉTODO}

Trata-se de um estudo de natureza quantitativa, tipo descritivo e exploratório, uma vez que foi desenvolvido e avaliado um Wiki para acesso à informação sobre os cuidados de Enfermagem para o paciente em ventilação mecânica em terapia intensiva. Fundamentado em bibliografias de referência, buscou-se, ainda, refletir sobre as opiniões dos participantes em relação à ferramenta tecnológica Wiki a partir das três questões abertas contidas no instrumento de avaliação. $\mathrm{O}$ estudo foi conduzido no mês de novembro de 2009.

O Wiki sobre ventilação mecânica em UTI foi desenvolvido pelas pesquisadoras dentro da plataforma MOODLE ${ }^{\circledR}$, sendo esta uma ferramenta tecnológica educacional disponibilizada aos alunos pela Universidade Federal de Santa Catarina (UFSC).

O MOODLE ${ }^{\circledast}$ é um Ambiente Virtual de Aprendizagem (AVA) que se tornou muito popular entre os educadores de todo mundo por ser uma ferramenta disponível para a criação de sites de Web dinâmicos para seus alunos. O foco do MOO$\mathrm{DLE}^{\circledast}$ é sempre proporcionar aos educadores as melhores ferramentas para gerir e promover a aprendizagem. Entretanto, este AVA propicia diversas maneiras de utilização, tais como: a) plataforma para realização de cursos totalmente à distância (online) ou para ferramenta de apoio para cursos presenciais; b) módulos de atividades como fóruns, Wikis e banco de dados para a criação de comunidades ricas de aprendizagem colaborativa em torno de um assunto/conteúdo (perspectiva do construtivismo social); c) disponibilização de conteúdos aos alunos e; d) avaliação da aprendizagem por meio de atribuições/atividades ou questionários ${ }^{(11)}$.

O desenvolvimento do Wiki abordou os seguintes tópicos: ventilação mecânica não invasiva (conceito; modalidades; vantagens e desvantagens; indicações, contraindicações e complicações; cuidados de enfermagem) e ventilação mecânica invasiva (conceito e objetivos; indicações; terminologia; modalidades; complicações; desmame ventilatório; cuidados de enfermagem).
Neste WIKI foi elaborado um texto descritivo dos itens de cada tópico mencionado e inseridos links de artigos científicos, imagens e vídeos referente ao tema ventilação mecânica.

No Wiki, por ser uma ferramenta colaborativa de acesso à informação e de construção coletiva do conhecimento, o texto descritivo, links, imagens e vídeos postados pelas pesquisadoras, podiam ser editados e/ou modificados e/ou acrescidos de novas informações/links/imagens/vídeos pelos acadêmicos de Enfermagem que participaram do estudo.

A amostra foi intencional (ou proposital), não probabilística, por julgamento, sendo baseada no pressuposto de que o conhecimento do pesquisador sobre a população e seus elementos pode ser utilizado para selecionar a população a ser incluída na amostra. Assim, selecionou-se propositalmente, o grupo de seis acadêmicos de Enfermagem que estavam na $7^{a}$ fase do Curso de Graduação em Enfermagem da Universidade Federal de Santa Catarina (UFSC) e em estágio curricular na Unidade de Terapia Intensiva. O critério para inclusão dos participantes no estudo foi ser aluno do Curso de Graduação em Enfermagem da UFSC, regularmente matriculado na disciplina "Cuidado de Enfermagem ao Indivíduo adulto em Condição Crítica de Saúde" (7ª fase).

Para a realização deste estudo foram respeitados todos os preceitos éticos determinados pela Resolução n¹96/96 do Conselho Nacional de Saúde através do cumprimento das exigências do Termo de Consentimento Livre e Esclarecido (TCLE), ou seja, o direito de informação do indivíduo e respeito à liberdade dos participantes para que possam, a qualquer momento, desistir do estudo. Por se tratar de uma pesquisa envolvendo seres humanos foram também adotados os cinco referencias básicos da Bioética, quais sejam: autonomia, não maleficência, beneficência, justiça e anonimato. Este estudo foi submetido e aprovado pelo Comitê de Ética em Pesquisa (CEP) da Universidade Federal de Santa Catarina (protocolo n 528/2009). Assim, em respeito às questões éticas, os participantes foram identificados como: A1, A2, A3, A4, A5 e A6.

Neste estudo foi elaborado um formulário eletrônico desenvolvido na ferramenta Survey Monkey ${ }^{\oplus}$ disponível no link: $<$ http://pt.surveymonkey.com/home.aspx $>$. Este instrumento de coleta de dados, fundamentado na International Standards Organization (ISO) 9241-11 ${ }^{(12)}$, avaliou os critérios de Ergonomia e de Usabilidade da ferramenta Wiki como tecnologia de acesso à informação sobre ventilação mecânica em UTI.

$\mathrm{Na}$ avaliação do critério Ergonomia, quatro categorias foram avaliadas: organização (três itens); interface (quatro itens); conteúdo (dois itens) e; técnico (dois itens). A ergonomia compreende as categorias organização, interface, técnico e conteúdo do sistema. O conteúdo, a partir da estruturação dos dados, é a capacidade do sistema atender adequadamente as necessidades do cliente e estabelecer a assistência mais eficaz visando sua recuperação ${ }^{(12)}$.

No critério de Usabilidade da ferramenta Wiki foram avaliados sete itens. A usabilidade mede a eficácia, a eficiência e a satisfação com que um usuário pode realizar um conjunto específico de tarefas em um ambiente particular; dentre seus objetivos destacam-se o de avaliar se tarefas simples e básicas são fáceis de serem desempenhadas pelos usuários. Ou seja, 
um sistema é considerado eficaz quando permite que os usuários atinjam seus objetivos ${ }^{(12)}$.

Para o processamento e análise dos dados quantitativos se utilizou a estatística descritiva envolvendo cálculos de tendência central (média), desvio padrão, valor máximo e valor mínimo. A estatística inferencial (variância) foi utilizada para o estabelecimento da significação estatística mediante a comparação entre a média pré-determinada - média alvo "4" (MUITO BOM) - e as médias obtidas em cada critério avaliado. Este último teste foi realizado visando sustentar com maior densidade as avaliações tomadas como uma tendência do grupo devido ao número reduzido de participantes. Considerou-se o nível de significância $\mathrm{P}<0.05$ para um intervalo de confiança de $95 \%$ entre os dados encontrados.

Os itens de avaliação foram distribuídos em uma escala de valores com as seguintes categorias de respostas: [5] Excelente; [4] Muito Bom; [3] Bom; [2] Regular; [1] Ruim. Considerou-se na avaliação que os valores das médias entre: 1 a 1,5 receberiam classificação [RUIM]; 1,51 a 2,5 [REGULAR]; 2,51 a 3,5 [BOM]; 3,51 a 4,5 [MUITO BOM]; e 4,51 a 5 [EXCELENTE].

\section{RESULTADOS E DISCUSSÕES}

A Tabela 1 apresenta a avaliação da categoria "Organização" do critério Ergonomia do Wiki sobre ventilação mecânica em UTI.
Observa-se que na avaliação Ergonômica da categoria "Organização" a média geral de 4,77 $( \pm 0,09)$ demonstra que os avaliadores consideraram a organização do Wiki como EXCELENTE, em torno da média geral. A análise do Pvalor geral $(0,0033)$ constatou que não houve diferença entre as avaliações realizadas pelos acadêmicos, uma vez que $P<0,05$. A avaliação positiva da Ergonomia referente à categoria Organização pode também ser afirmada por meio das opiniões de alguns avaliadores.

A acessibilidade é um fator bem positivo e a linguagem é clara e fácil (A1).

Facilidade de acesso e navegação, clareza das informações [...] (A3).

O termo Wiki (do havaiano "wiki-wiki" = rápido, veloz, célere) foi criado por Ward Cunningham e disponibilizado em 1995 na web. Este autor tinha como objetivo desenvolver um site onde os próprios usuários poderiam gerar conteúdo ${ }^{(13)}$, ou ainda acessar informações de forma rápida e em tempo real.

A partir desse novo conceito de geração de conteúdo/conhecimento, o sistema Wiki disponibilizado pela Web 2.0, se diferencia das outras páginas da internet, uma vez que o conteúdo pode ser organizado, editado e atualizado constantemente pelos usuários sem haver a necessidade de autorização

Tabela 1 - Avaliação Ergonômica - Categoria Organização

\begin{tabular}{|c|c|c|c|c|c|c|c|c|c|c|c|}
\hline \multirow{2}{*}{$\begin{array}{l}\text { Itens de Avaliação } \\
\text { ORGANIZAÇÃO }\end{array}$} & \multicolumn{6}{|c|}{ Avaliadores } & \multirow[t]{2}{*}{ Média } & \multirow[t]{2}{*}{ Dpad } & \multirow[t]{2}{*}{ máx } & \multirow[t]{2}{*}{ mín } & \multirow[t]{2}{*}{ Pvalor } \\
\hline & $\mathrm{A} 1$ & $\mathrm{~A} 2$ & $\mathrm{~A} 3$ & A4 & A5 & A6 & & & & & \\
\hline $\begin{array}{l}\text { Organização e modo de acesso aos links do } \\
\text { WIKI funcionam adequadamente }\end{array}$ & 4 & 5 & 5 & 4 & 5 & 5 & 4,66 & 0,51 & 5 & 4 & 0,26666 \\
\hline O WIKI possui facilidade de operacionalização & 5 & 5 & 5 & 4 & 5 & 5 & 4,83 & 0,40 & 5 & 4 & 0,16666 \\
\hline $\begin{array}{l}\text { O WIKI é uma ferramenta tecnológica de } \\
\text { suporte para acesso à informação e aprendizagem }\end{array}$ & 5 & 5 & 5 & 4 & 5 & 5 & 4,83 & 0,40 & 5 & 4 & 0,16666 \\
\hline MÉDIA & & & & & & & 4,77 & & & & \\
\hline DESVIO PADRÃO & & & & & & & 0,09 & & & & \\
\hline Pvalor GERAL & & & & & & & 0,0033 & & & & \\
\hline
\end{tabular}

Tabela 2 - Avaliação Ergonômica - Categoria Interface

\begin{tabular}{|c|c|c|c|c|c|c|c|c|c|c|c|}
\hline \multirow{2}{*}{$\frac{\text { Itens de Avaliação }}{\text { INTERFACE }}$} & \multicolumn{6}{|c|}{ Avaliadores } & \multirow[t]{2}{*}{ Média } & \multirow[t]{2}{*}{ Dpad } & \multirow[t]{2}{*}{ máx } & \multirow[t]{2}{*}{ mín } & \multirow[t]{2}{*}{ Pvalor } \\
\hline & A1 & $\mathrm{A} 2$ & A3 & A4 & A5 & A6 & & & & & \\
\hline A interface do Wiki para uso é atrativa (aparência das telas) & 4 & 5 & 4 & 4 & 5 & 5 & 4,5 & 0,54 & 5 & 4 & 0,3 \\
\hline $\begin{array}{l}\text { O Wiki possui estrutura lógica dos dados: como as informações } \\
\text { aparecem ao usuário (são compreensíveis) }\end{array}$ & 4 & 5 & 4 & 4 & 5 & 5 & 4,5 & 0,54 & 5 & 4 & 0,3 \\
\hline $\begin{array}{l}\text { A quantidade de informação é suficiente para a assistência de } \\
\text { enfermagem ao paciente em ventilação mecânica na UTI }\end{array}$ & 5 & 4 & 5 & 3 & 4 & 5 & 4,33 & 0,81 & 5 & 4 & 0,66666 \\
\hline O Wiki possui conforto visual para manuseio dos links e das telas & 5 & 5 & 5 & 5 & 5 & 5 & 5 & 0 & 5 & 5 & 0 \\
\hline \multicolumn{7}{|l|}{ MÉDIA } & 4,58 & & & & \\
\hline \multicolumn{7}{|l|}{ DESVIO PADRÃO } & 0,28 & & & & \\
\hline \multicolumn{7}{|l|}{ Pvalor GERAL } & 0,0744 & & & & \\
\hline
\end{tabular}


do autor da versão anteriormente elaborada. Esta ferramenta permite, no sentido de construção coletiva do conhecimento, corrigir erros e inserir novas informações, ou seja, ninguém é autor proprietário de nenhum texto e o seu conteúdo pode ser sempre atualizado devido à possibilidade de ser reformulado por outro usuário ${ }^{(14)}$.

A avaliação Ergonômica da categoria Interface é apresentada na Tabela 2.

Observa-se que a avaliação Ergonômica da categoria "Interface" obteve média geral de 4,58 $( \pm 0,28)$, constatando que os avaliadores consideraram a interface do Wiki como EXCELENTE. A análise do nível de significância constatou que somente no item "o Wiki possui conforto visual para o manuseio dos links e telas" não houve diferença entre as avaliações dos acadêmicos de enfermagem, pois o Pvalor foi <0,05 (0). O Pvalor geral obtido $(0,0744)$ evidenciou que houve diferença significativa na avaliação dos participantes do estudo, pois Pvalor $>0,05$. A opinião do acadêmico A4 reforça os dados obtidos.

A interface está objetiva, com quantidade de informações suficientes [...], entretanto sugiro inserir mais links de acesso a vídeos autoexplicativos sobre ventilação mecânica (A4).

Ao planejar e desenvolver a interface do Wiki no ambiente MOODLE ${ }^{\circledR}$, levou-se em consideração o desenho, as cores, as imagens e o arranjo das informações ${ }^{(15)}$, propiciando aos avaliadores uma aparência atrativa das telas, uma estruturação lógica dos dados, bem como, o conforto visual necessário e desejável do texto descritivo, das imagens e dos links de acesso às informações.

A ferramenta Wiki possibilita a conexão e a interação dos usuários por meio da criação de uma comunidade de pessoas com interesses comuns. Esta ferramenta, por utilizar a WEB como plataforma e por adotar a arquitetura da participação e do aproveitamento da inteligência coletiva como competências chaves, apresenta algumas características específicas, tais como: modelos leves de programação; experiência e conhecimento próprio dos usuários; confiança nos seus usuários; ênfase nos usuários e não na tecnologia; o comportamento do usuário não está pré-determinado e; o software melhora na medida que mais pessoas utilizam ${ }^{(8)}$.

A Tabela 3 apresenta a avaliação da categoria "Conteúdo" do critério Ergonomia do Wiki sobre ventilação mecânica em UTI.
A partir dos valores atribuídos aos dois itens da categoria "Conteúdo", do critério de Ergonomia e da média geral de $4,75( \pm 0,11)$ observa-se que os avaliadores consideraram o conteúdo adicionado/postado na ferramenta Wiki como EXCELENTE, em torno da média geral. A análise do Pvalor geral $(0,0022)$ constatou que não houve diferença entre as avaliações realizadas pelos acadêmicos, uma vez que $\mathrm{P}<0,05$. Os relatos abaixo reforçam os resultados positivos referentes à avaliação do conteúdo disponibilizado na ferramenta Wiki.

[...] facilita muito para quem tem pouco tempo para retomar alguns conteúdos necessários para prestar uma assistência de qualidade ao paciente que necessita de cuidados críticos (A6).

O conteúdo está abrangente e completo, permitindo acessar informações importantes para o cuidado de enfermagem em tempo real (A2).

A informação é um elemento central para o processo de decisão e um requisito fundamental para a gestão e prestação qualificadas da atenção à saúde. $\mathrm{O}$ acesso à informação é reconhecido como aspecto fundamental para o planejamento, o funcionamento, a supervisão e o controle dos programas assistenciais da saúde ${ }^{(16)}$.

Os profissionais de saúde se destacam entre os que mais necessitam e utilizam a informação para o desempenho de suas atividades e que as mesmas são diretamente influenciadas pelo acesso e pela qualidade da informação que utilizam, o que pode acarretar sérias consequências para os pacientes e até mesmo para a sociedade com um todo ${ }^{(17)}$.

Nesta perspectiva, o Wiki, caracterizado pela sua interatividade e flexibilidade de utilização, foi desenvolvido visando contemplar todo o conteúdo necessário sobre ventilação mecânica em UTI para que o acadêmico de Enfermagem tivesse disponível uma importante fonte de informação e conhecimento de acesso fácil, rápido e seguro. Ressalta-se, entretanto, que por se tratar de uma ferramenta que também é colaborativa, ou seja, que permite a construção coletiva do conhecimento, os participantes do estudo foram orientados sobre a possibilidade de contribuir com novas informações por meio de novas edições/atualizações e inserção de novos links, imagens e vídeos.

Tabela 3 - Avaliação Ergonômica - Categoria Conteúdo

\begin{tabular}{|c|c|c|c|c|c|c|c|c|c|c|c|}
\hline Itens de Avaliação & \multicolumn{6}{|c|}{ Avaliadores } & Média & Dpad & máx & mín & Pvalor \\
\hline CONTEÚDO & A1 & A2 & A3 & A4 & A5 & A6 & & & & & \\
\hline As informações são claras, objetivas e atualizadas & 5 & 5 & 5 & 4 & 5 & 5 & 4,83 & 0,40 & 5 & 4 & 0,33333 \\
\hline $\begin{array}{l}\text { O conteúdo está inter-relacionado e consistente } \\
\text { com a área da terapia intensiva }\end{array}$ & 5 & 5 & 4 & 4 & 5 & 5 & 4,66 & 0,51 & 5 & 4 & 0,26666 \\
\hline MÉDIA & & & & & & & 4,75 & & & & \\
\hline DESVIO PADRÃO & & & & & & & 0,11 & & & & \\
\hline Pvalor GERAL & & & & & & & 0,0022 & & & & \\
\hline
\end{tabular}


Outras características se destacam nesta nova geração de ferramentas da tecnologia WEB 2.0, tais como: a) a internet como plataforma para processar, produzir ou consumir informação, onde um computador conectado a ela é ferramenta básica e principal de trabalho; b) valorização do conteúdo colaborativo e da inteligência coletiva, onde o conteúdo deve ser produzido e consumido por qualquer pessoa de forma simples e direta e; c) permite que usuários comuns, que até então não possuíam conhecimentos necessários para publicar conteúdo na Internet - pela ausência de ferramentas de uso simplificado - publicassem e consumissem informação de forma rápida e constante. Notadamente têm-se os Wikis como expoentes desta massificação(18). A avaliação Ergonômica da categoria "Técnico" da ferramenta Wiki é apresentada na Tabela 4.

Observa-se que a categoria "Técnico" do critério Ergonomia obteve média geral de 4,66 $( \pm 0)$ revelando que os avaliadores consideraram o componente técnico do Wiki como EXCELENTE em torno da média geral. A análise do Pvalor geral
(0) constatou que não houve diferença entre as avaliações realizadas pelos acadêmicos, uma vez que $\mathrm{P}<0,05$.

Ao estruturar e disponibilizar as informações sobre ventilação mecânica em UTI numa ferramenta da Web 2.0 que funciona adequada e satisfatoriamente se acredita que esta tecnologia emergente pode contribuir com o aprimoramento do pensamento crítico e do julgamento clínico dos futuros enfermeiros.

O pensamento crítico, considerado um julgamento intencional, auto-regulatório, resultante da interpretação, análise, avaliação e inferência e o julgamento/raciocínio clínico, característica essencial para promover a melhoria da prática assistencial(19), podem ser aprofundados e apoiados pela ferramenta Wiki.

A Tabela 5 apresenta os sete itens avaliados no critério de Usabilidade do Wiki sobre ventilação mecânica em UTI.

Observa-se na Tabela 5 que, na avaliação de Usabilidade, a média geral atribuída pelos avaliadores foi de 4,71 $( \pm 0,20)$, demonstrando que os acadêmicos de Enfermagem

Tabela 4 - Avaliação Ergonômica - Categoria Técnico

\begin{tabular}{l|c|c|c|c|c|c|c|c|c|c|c|}
\hline \multicolumn{1}{c|}{ Itens de Avaliação } & \multicolumn{5}{c|}{ Avaliadores } & Média & Dpad & máx & mín & Pvalor \\
\hline \multicolumn{1}{c|}{ TÉCNICO } & A1 & A2 & A3 & A4 & A5 & A6 & & & & \\
\hline $\begin{array}{l}\text { As informações estruturadas no WIKI permitem raciocínio com- } \\
\text { patível com a clínica de enfermagem em ventilação mecânica }\end{array}$ & 4 & 5 & 5 & 4 & 5 & 5 & 4,66 & 0,51 & 5 & 4 & 0,26666 \\
O WIKI funciona adequadamente & 4 & 5 & 5 & 4 & 5 & 5 & 4,66 & 0,51 & 5 & 4 & 0,26666 \\
MÉDIA & & & & & \\
DESVIO PADRÃO \\
Pvalor GERAL
\end{tabular}

Tabela 5 - Avaliação de Usabilidade do Wiki.

\begin{tabular}{|c|c|c|c|c|c|c|c|c|c|c|c|}
\hline Itens de Avaliação & \multicolumn{6}{|c|}{ Avaliadores } & \multirow[t]{2}{*}{ Média } & \multirow[t]{2}{*}{ Dpad } & \multirow[t]{2}{*}{ máx } & \multirow[t]{2}{*}{ mín } & \multirow[t]{2}{*}{ Pvalor } \\
\hline INTERFACE & A1 & A2 & A3 & A4 & A5 & A6 & & & & & \\
\hline As telas do sistema são claras, fáceis de ler e interpretar & 5 & 5 & 5 & 4 & 5 & 5 & 4,83 & 0,40 & 5 & 4 & 0,16666 \\
\hline O usuário é capaz de acessar o Wiki facilmente & 5 & 5 & 5 & 5 & 5 & 5 & 5 & 0 & 5 & 4 & 0 \\
\hline A estrutura da informação disponibilizada é viável e fácil de usar & 5 & 5 & 5 & 4 & 5 & 5 & 4,83 & 0,40 & 5 & 4 & 0,16666 \\
\hline $\begin{array}{l}\text { O Wiki favorece o tratamento eficiente aos problemas de sua } \\
\text { dimensão no cuidado de enfermagem ao paciente em ventilação } \\
\text { mecânica em UTI }\end{array}$ & 4 & 5 & 5 & 3 & 5 & 5 & 4,5 & 0,83 & 5 & 4 & 0,7 \\
\hline $\begin{array}{l}\text { O Wiki é uma ferramenta colaborativa (você pode contribuir } \\
\text { com as informações) }\end{array}$ & 4 & 5 & 5 & 3 & 5 & 5 & 4,5 & 0,83 & 5 & 4 & 0,7 \\
\hline $\begin{array}{l}\text { O Wiki permite um manejo eficiente das informações que utiliza } \\
\text { e pode ser ampliado }\end{array}$ & 4 & 5 & 5 & 3 & 5 & 5 & 4,5 & 0,83 & 5 & 4 & 0,7 \\
\hline $\begin{array}{l}\text { O Wiki roda em qualquer navegador (Firefox, Explorer, Netscape) } \\
\text { no ambiente MOODLE }{ }^{\circledR}\end{array}$ & 5 & 5 & 5 & 4 & 5 & 5 & 4,83 & 0,40 & 5 & 4 & 0,16666 \\
\hline \multicolumn{7}{|l|}{ MÉDIA } & 4,71 & & & & \\
\hline \multicolumn{7}{|l|}{ DESVIO PADRÃO } & 0,20 & & & & \\
\hline \multicolumn{7}{|l|}{ P valor GERAL } & 0,0979 & & & & \\
\hline
\end{tabular}


consideraram a usabilidade da ferramenta Wiki como EXCELENTE. A análise do nível de significância constatou que somente no item "o usuário é capaz de acessar o Wiki facilmente" não houve diferença entre as avaliações dos acadêmicos de enfermagem, pois o Pvalor foi <0,05 (0). O Pvalor geral obtido $(0,0979)$ evidenciou que houve diferença significativa na avaliação dos participantes do estudo, pois Pvalor >0,05. Entretanto, as falas dos participantes do estudo reforçam os resultados positivos obtidos em torno da média alvo "4" referentes à avaliação da usabilidade da ferramenta Wiki como tecnologia de acesso à informação.

Excelente. Oportuniza que capacitações possam ser realizadas de dentro do ambiente de trabalho, sem horário e local determinados. Facilita acesso ao curso e permite que cada usuário faça uso da ferramenta de acordo com seu próprio ritmo de estudo (A5).

Acredito que esses ambientes virtuais contribuem muito para o aprendizado e também, por ser uma ferramenta online, facilita o acesso à informação. É uma forma rápida de fazer consultas também (A1).

Ele (WIKI) serve como um facilitador na compreensão de conteúdos complexos por utilizar uma linguagem mais "palpável". A forma que as informações foram colocadas também ajuda muito no entendimento do tema estudado (A2).

É uma ferramenta de fácil manuseio, de acesso rápido, que possibilita acrescentar conhecimentos além dos oferecidos, sendo, portanto, uma ferramenta importante para o enriquecimento do aprendizado para cuidados intensivos (A6).

Eu gostei muito, pois dessa forma a informação é de acesso rápido e eu posso contribuir com a construção do conhecimento também (A4).

Os Wikis apresentam alguns princípios, tais como: ferramenta "aberta"; incrementado; orgânico; universal; preciso; tolerante; observável; convergente; confiável; divertido e; compartilhável ${ }^{(13)}$.

Os Wikis são considerados ferramentas abertas onde qualquer usuário pode ter acesso a página, podendo alterar seu conteúdo quando considerar que esteja incompleto ou mal organizado, editar e/ou criar uma nova página. Esta ferramenta também é incremental, pois as páginas podem apresentar links para outras páginas do próprio Wiki, inclusive para páginas que ainda não foram escritas e ainda ser orgânico por permitir a edição e evolução da organização estrutural do site e dos textos ${ }^{(13)}$.

Os Wikis são universais porque os mecanismos da edição e de organização são os mesmos, de modo que todo usuário é automaticamente um organizador e editor. Esta tecnologia é precisa uma vez que os nomes das páginas são precisos o suficiente para evitar conflitos de nomes, tolerante, pois o comportamento interpretativo é preferido às mensagens de erro e ainda, observável, à medida que as atividades desenvolvidas no site podem ser observadas e revisadas por todos ${ }^{(13)}$.
O Wiki é convergente no sentido de que a duplicação de páginas similares não é desejável e podem ser redirecionadas ou removidas, se caracterizada pela confiança uma vez que se torna necessário confiar nos usuários e no processo de evolução contínua dos conteúdos postados/editados. Outros dois princípios são o divertimento, pois se entende que a melhor participação e envolvimento das pessoas ocorrem quando a interação é feita de forma mais espontânea e prazerosa do que por obrigação e, por fim, o compartilhamento se concretizando como um espaço de acesso e troca de informações, conhecimentos, experiências e idéias ${ }^{(13)}$.

Além dos princípios estabelecidos, algumas características básicas da ferramenta Wiki se destacam, entre elas: a) software livre de fácil instalação e compatível com as plataformas Linux e Windows; b) permite discussão assíncrona; c) permite importação e exportação de textos e imagens facilitando a criação automática de hipertexto e hiperlinks; d) não existe qualquer mecanismo de revisão preliminar à publicação, portanto a responsabilidade pela qualidade das contribuições é de cada participante autorizado; e) a autorização para contribuir no sistema pode ser programada pelo grupo gestor, podendo ser ampla e irrestrita ou possuir algumas restrições como, por exemplo, estar cadastrado ${ }^{(20)}$.

As possibilidades de utilização da ferramenta Wiki remetem à ideia de conexão entre professor e aluno. Esta conectividade ocorre por meio da possibilidade de alteração do conhecimento anteriormente gerado, criando assim um novo espaço de aprendizagem contínua através da troca e aquisição de novas informações ${ }^{(8)}$.

Constata-se que a conexão e a interação, características essenciais e transformadoras para o processo de ensino-aprendizagem vão ao encontro do funcionamento da ferramenta Wiki, uma vez que: a) a aprendizagem e o conhecimento é um processo de conectar nós especializados ou fontes de informação, que pode residir em dispositivos não humanos e que se apoia na diversidade de opiniões; b) a capacidade de saber mais é mais crítica do que aquilo que é conhecido atualmente; c) há a necessidade de cultivar e manter conexões para facilitar a aprendizagem contínua; d) a habilidade de enxergar conexões entre áreas, ideias e conceitos é uma habilidade fundamental; e) a atualização ("currency" - conhecimento acurado e em dia) é a intenção de todas as atividades de aprendizagem conectivistas e; f) a tomada de decisão é, por si só, um processo de aprendizagem ${ }^{(21)}$.

Escolher o que aprender e o significado das informações que chegam ao usuário é enxergar através das lentes de uma realidade em mudança. Apesar de haver uma resposta certa agora, ela pode ser errada amanhã devido a mudanças nas condições que cercam a informação e que afetam a decisão(21).

\section{CONCLUSÕES}

A informática, especialmente a ferramenta Wiki, disponibilizada pelas Tecnologias da Web 2.0, tem propiciado possibilidades de mudanças e contribuído de forma crescente e cada vez mais acelerada nos cenários que envolvem o processo de ensino-aprendizagem. 
Entende-se que as novas tecnologias disponibilizadas pela área da informática podem aprimorar e fortalecer o processo de ensino-aprendizagem dos acadêmicos de Enfermagem no tocante aos cuidados intensivos realizadas nos cenários das UTIs. Por conseguinte, os alunos ao adotar a informática como ferramenta racional da sua assistência, poderão adquirir maior conhecimento científico e realizar a assistência integral ao paciente, associando adequadamente a teoria e a prática.

As avaliações quantitativas e qualitativas dos acadêmicos de Enfermagem permitem concluir que a ferramenta Wiki possui qualidade e efetividade como um todo, uma vez que os critérios de Ergonomia (organização, interface, conteúdo e técnico) e Usabilidade foram avaliados como excelentes pelos participantes.

Assim, é possível afirmar que o objetivo traçado para este estudo, foi alcançado com êxito, uma vez que a ferramenta Wiki foi considerada uma tecnologia coerente, efetiva, possível e consistente, pois permitiu o acesso à informação sobre ventilação mecânica em UTI de forma rápida, fácil e segura em tempo real.

Nesta perspectiva de interação entre os novos paradigmas da educação, onde o aluno passa a ser o gestor do próprio processo de aprender, e as novas tecnologias emergentes disponibilizadas pela área da informática, as ferramentas da Web 2.0 ganham destaque nesse atual cenário educacional.

\section{REFERÊNCIAS}

1. Zuzelo PR, Gettis C, Hansell AW, Thomas L. Describing the influence of technologies on registered nurses' work. Clin Nurse Spec 2008;22(3):132-40.

2. Sasso GTMD, Barbosa SFF. Perspectivas futuras à informática em enfermagem: a aplicabilidade dos ambientes hipermídia no processo ensino-aprendizagem. Texto \& Contexto Enferm 2000;9(1):79-92.

3. Camacho ACLF. Análise das publicações nacionais sobre a educação à distância na enfermagem. Rev Bras Enferm 2009;62(4):588-93.

4. Peterlini OLG, Zagonel IPS. O sistema de informação utilizado pelo enfermeiro no gerenciamento do processo de cuidar. Texto \& Contexto Enferm 2006;15(3):418-26.

5. Thofehrn MB, Leopardi MT, Amestoy SC. Construtivismo: experiência metodológica em pesquisa na enfermagem. Acta Paul Enferm 2008;21(2):312-6.

6. Jonassen DH, Myers JM, Mckillop AM. From constructivism to constructionism: learning with hypermedia/ multimedia rather than from it. In: Wilson BG, editor. Constructivist learning environments: case studies in instructional design. Englewood Cliffs: Educational Technology Publications; 1996. p. 9-106.

7. Jonassen $\mathrm{DH}$. O uso das novas tecnologias na educação a distância e a aprendizagem construtivista. Aberto [periódico na internet]. 1996 [acesso em 17 set 2009]16;(70):7088 Disponível em: < http://www.rbep.inep.gov.br/index. php/emaberto/article/viewFile/1054/956>

8. Silva FCC. Avaliação de fontes de informação na Web: um estudo focado na Wikipédia. Florianópolis. Dissertação [Mestrado em Ciência da Informação]- Universidade Federal de Santa Catarina; 2008.

9. Fernandes GG. Novos desafios em ambientes virtuais de ensino e aprendizagem (AVEA). Universidade Federal do Piauí (Programa de educação à distância); 2006.

10. Prado C, Martins CP, França L, Leite MMJ, Peres HHC. Metodologia de utilização do chat na enfermagem. Rev Bras Enferm 2009;62(4):594-8.

11. MOODLE. [homepage na internet]. What is Moodle? [acesso em 23 set 2009] Disponível em: < http://moodle. org/about/ $>$.

12. International Standars Organization/International Electrotechnical Commission ISO/IEC 9241-11 - Usability Net. 2006 [acesso 17 jul 2007]. Disponível em: < http://www. usabilitynet.org/tools/r_international.htm $>$.

13. Wiki design principles [homepage na internet]. Wiki design principles. [acesso em 21 ago 2009] Disponível em: < http://c2.com/cgi/wiki?WikiDesignPrinciples > .

14. Rupley S. What's a Wiki? In: PC Magazine [homepage na internet]. [acesso em 21 ago 2009] Disponível em: < http:// www.pcmag.com/article2/0,4149,1071705,00.asp > .

15. DiMarco J. Web portfolio design and application. Hershey: Idea Group Publishing; 2006.

16. Organización Panamericana de la Salud. Desarrollo de sistemas normalizados de información de enfermería. Washington: OPS; 2001.

17. Barbosa SFF, Sasso GTMD. Internet e saúde: um guia para os profissionais. Blumenau: Nova Letra; 2007.

18. O'Reilly T. [homepage na internet]. What is web 2.0: design patterns and business models for the next generation of software. [acesso em 20 ago 2009]. Disponível em: < http://www.oreillynet.com/pub/a/oreilly/tim/ news/2005/09/30/what-is-web-20.html >

19. Roza BA. Pensamento crítico e julgamento clínico na enfermagem. In: Bork AMT. Enfermagem baseada em evidências. Rio de Janeiro: Guanabara Koogan; 2005. p. 95-111.

20. Faquetti MF, Alves MBM. Wikis e o bibliotecário de referência: novos ambientes de aprendizagem. In: Seminário Nacional de Bibliotecas Universitárias [evento na internet]. 2006 dez 14; Salvador, Brasil [acesso em 20 ago 2009] Disponível em: <http://www.snbu2006.ufba.br/ soac/viewabstract.php?id $=333>$.

21. Siemens G. Uma teoria de aprendizagem para a Idade Digital. Competências profissionais In: Webcompetencias [homepage na internet]. [acesso em 21 ago 2009] Disponível em: < http://www.webcompetencias.com/textos/ conectivismo.htm $>$. 